\title{
K-SEMISTABILITY FOR IRREGULAR SASAKIAN MANIFOLDS
}

\author{
TRISTAN C. COLLINS AND GÁBOR SZÉKELYHIDI
}

\begin{abstract}
We introduce a notion of K-semistability for Sasakian manifolds. This extends to the irregular case the orbifold K-semistability of Ross-Thomas. Our main result is that a Sasakian manifold with constant scalar curvature is necessarily K-semistable. As an application, we show how one can recover the volume minimization results of Martelli-Sparks-Yau, and the Lichnerowicz obstruction of GauntlettMartelli-Sparks-Yau from this point of view.
\end{abstract}

\section{INTRODUCTION}

Determining necessary and sufficient conditions for the existence of constant scalar curvature Kähler ( $\operatorname{cscK}$ ) metrics on a compact Kähler manifold $X$ is an important open problem in complex geometry, requiring methods from algebraic geometry and partial differential equations. When $c_{1}(X)$ represents a negative or trivial cohomology class then Yau [40] proved that there exist Kähler-Einstein metrics in classes proportional to $c_{1}(X)$ (see also Aubin [3] for the $c_{1}(X)<0$ case). However when the first Chern class is postive, or we are looking at general Kähler classes, then there are obstructions to existence. A famous conjecture of Yau says that the existence of Kähler-Einstein metrics, when $c_{1}(X)>0$, should be equivalent to some geometric invariant theory (GIT) notion of stability for the underlying variety [41. Tian [38] introduced the notion of K-stability, and showed that it is a necessary condition for existence. The notion of K-stability was refined by Donaldson [10], and extended to any Kähler class given by the first Chern class $c_{1}(L)$ of an ample line bundle $L$. The Yau-Tian-Donaldson conjecture states that K-stability (or some refinement of it) of a polarized manifold $(X, L)$ is equivalent to the existence of a $\operatorname{cscK}$ metric in $c_{1}(L)$. For more on this very active area of research, see the survery of Phong-Sturm [28], and the references therein.

From the works of Donaldson [9], Stoppa [36] and Mabuchi [23] we know that the existence of a cscK metric implies K-stability. Part of this work was subsequently generalized by Ross-Thomas [32 to the case of orbifolds with cyclic quotient singularities, where they showed that the existence of a cscK orbifold metric in $c_{1}(L)$ implies the K-semistability of the polarized orbifold $(X, L)$. As we will recall below, the work of Ross-Thomas can be 
phrased as a result about quasi-regular Sasakian manifolds, and the goal of the present paper is to extend this to the irregular case.

Sasakian geometry is an important, odd-dimensional conterpart of Kähler geometry. Recently, the subject of Sasakian geometry has garnered a great deal of interest partly due to its connections to the AdS/CFT correspondence in theoretical physics [24], which provides a detailed correspondence between certain conformal field theories and geometries (see also [19, 20, 1, 27]). It is thus a natural problem to determine necessary and sufficient conditions for the existence of Sasaki-Einstein metrics, and more generally, Sasakian metrics of constant scalar curvture (or even the analogs of extremal metrics [5]). As in the Kähler case, this is well understood when the basic first Chern class is negative or zero [11, 34]. However, when the basic first Chern class is positive, there are obstructions to existence [15, 16, 25]. It is expected that a suitably generalized version of the conjecture of Yau [4] should hold. Some sufficient conditions for the existence of Sasaki-Einstein metrics have been provided in [8] in the spirit of [29, 30, by examining conditions which are sufficient to guarantee the convergence of the Sasaki-Ricci flow, introduced in [34]. However, these conditions are not obviously GIT related. Recent work on obstructions to the existence of Sasaki-Einstein metrics has provided the first hints of a connection to stability for Sasakian manifolds; see, for example, [25, 26] and the references therein. In [25] the authors show that a Sasakian manifold admits a Sasaki-Einstein metric only when the Reeb vector field minimizes the volume functional. Moreover, when the minimizing Reeb vector field is quasi-regular, they show that volume minimization is equivalent to the vanishing of the classical Futaki invariant on the quotient orbifold. This is a remarkable result, translating a problem which seems algebro-geometric in nature into a dynamical problem which can be studied analytically.

In the current work, our primary interest is to develop a notion of Kstability for a Sasakian manifold $S$. When the Sasakian manifold is quasiregular, then this is equivalent to the work of Ross-Thomas [32] on Kstability for orbifolds. The question of whether there is a suitable extension of this to the irregular case has been posed in several places in the literature [35. Problem 7.1], [25, 13. In this paper we provide such an extension, and prove that the existence of a constant scalar curvature Sasakian metric implies the K-semistability of $S$. Our main result is thus the following corollary of Theorem 5 .

Corollary 1. Let $(S, g)$ be a Sasakian manifold with Reeb vector field $\xi$. If $g$ has constant scalar curvature, then the cone $(C(S), \xi)$ is K-semistable.

As already suggested by Sparks [35], the obvious approach is to approximate a given irregular Sasakian manifold with a sequence of regular ones, and attempt to take a limit of the obstructions provided by the results of Ross-Thomas in the orbifold case. The main difficulty with this is that when the Reeb vector field is irrational, then it is not clear how to generalize the 
usual setup of K-stability to the non-Hausdorff quotient space. If instead we were to work directly with the odd dimensional Sasakian manifolds, then the methods of algebraic geometry are no longer available to define testconfigurations and their Futaki invariants. Because of this we work on the cone over the Sasakian manifold, which is an affine variety with an isolated singular point. These affine varieties come with the action of a torus $T$, and a Reeb field on the Sasakian manifold corresponds to an element in the Lie algebra of $T$. We can naturally define test-configurations for affine varieties with a $T$-action, and for any Reeb field $\xi$ on the underlying Sasakian manifold we define a Futaki invariant of such a test-configuration. The crucial points are that this Futaki invariant (for a given test-configuration) varies continuously with the Reeb field $\xi$, and when $\xi$ is rational, then it matches up with Ross-Thomas's Futaki invariant for test-configurations in the orbifold case. We can then use an approximation argument to extend Ross-Thomas's results to the case of irrational Reeb fields.

Our definition of the Futaki invariant uses the Hilbert series as opposed to the usual Riemann-Roch expansions. This point of view was already used in 25] in the form of the index character, to compute the volume of a Sasakian manifold. The main advantage is that for orbifolds the RiemannRoch expansions contain periodic terms, which become unmanageable as we approach an irrational Reeb field, whereas in the index character these periodic terms are not visible. This allows us to show the continuity of the Futaki invariant with respect to the Reeb field.

As an application, we recover the results of [25] algebraically by showing that in the situation they considered, volume minimization is equivalent to K-semistability for product test configurations. As a second application, we show that the Lichnerowicz obstruction to existence of Sasaki-Einstein metrics studied in [16] can be interpreted in terms of K-semistability for deformations arising from the Rees algebra of a principal ideal of the coordinate ring. Indeed, for rational Reeb fields, the Lichnerowicz obstruction was interpreted in terms of slope stability for the quotient orbifold in 32 . Our computations recover this result, and extend it to irrational Reeb fields by establishing an explicit formula for the Donaldson-Futaki invariant of the Rees deformation. It is worth pointing out that both volume minimization and the Lichnerowicz obstruction has a natural interpretation in terms of the AdS/CFT dual field theory; see [26, 16] for details. This presents an interesting opportunity to attempt to recover the necessity of K-semistability for existence of Sasaki-Einstein metrics as the geometric dual of a physical constraint in the dual conformal field theory. Such a connection would be beneficial from both a physical and mathematical standpoint.

We begin our developments in section 2 with a brief review of Sasakian manifolds, and affine schemes polarized by a Reeb vector field. We also briefly recall some facts about orbifolds and orbifold K-stability . In section 3 we define the Calabi functional on a polarized affine variety equipped with a Kähler metric compatible with the Reeb field. In section 4 we discuss 
the index character and the Donaldson-Futaki invariant of a polarized affine variety. In section 4 we define test configurations for polarized affine varieties, and K-semistability. We then use the results of sections 2, 3 and 4 to prove our main theorem. Finally, in section 6 we show that the volume minimization results of Martelli, Sparks and Yau [25, and the Lichnerowicz obstruction of [16] arise from K-stability considerations for product test configurations, and the Rees algebra respectively.

Acknowledgements The first author would like to thank his advisor Professor D.H. Phong for his support and encouragement, as well as Zack Maddock, You Qi, and Michael McBreen for many helpful conversations. The second author would like to thank Professor S.T. Yau for introducing him to Sasakian manifolds, and J. Ross for helpful discussions on orbifold Kstability.

\section{BACKGROUND}

2.1. Sasakian geometry. In this section we will recall some aspects of Sasakian geometry that we will use. There are various points of view on the subject, and for a thorough treatment see Boyer-Galicki [7].

Definition 2.1. A Sasakian manifold is a smooth Riemannian manifold $(S, g), \operatorname{dim}_{\mathbb{R}} S=2 n+1$, such that the metric cone $(C(S), \bar{g}):=(S \times$ $\left.\mathbb{R}_{\geq 0}, d r^{2}+r^{2} g\right)$ is Kähler. Note that $S$ is canonically imbedded in $C(S)$ as the set $\{r=1\}$.

A Sasakian manifold inherits a number of key properties from its Kähler cone. In particular, an important role is played by the Reeb vector field.

Definition 2.2. The Reeb vector field is $\xi=\left.J\left(r \partial_{r}\right)\right|_{\{r=1\}}$, where $J$ denotes the integrable complex structure on $C(S)$.

The Reeb vector field is a unit length, real holomorphic, Killing vector field whose integral curves foliate $S$ by geodesics. Sasakian manifolds are roughly categorized by their Reeb vector fields. When the intregral curves of $S$ are all compact, the action of the Reeb vector field integrates to a $U(1)$ action. A Sasakian manifold is said to be regular if this $U(1)$ action is free; otherwise, it is said to be quasi-regular. When the integral curves of the Reeb vector field are not all compact, the Sasakian manifold is said to be irregular. The regular and quasi-regular Sasakian manifolds are well understood, owing to the following theorem of Boyer and Galicki.

Theorem 1 (6] Theorem 2.4). Let $S$ be a compact regular or quasi-regular Sasakian manifold. The space of leaves of the Reeb foliation $Z$ is a Kähler manifold or orbifold, respectively. Moreover, $Z$ is a normal, projective, $\mathbb{Q}$ factorial algebraic variety.

The results of Rukimbira 33 imply that any irregular Reeb vector field can be approximated by quasi-regular Reeb fields. In particular, every 
Sasakian manifold admits at least one quasi-regular Reeb vector field. Combining this with Theorem 1, we see that for any Sasakian manifold $S$, the cone over $S$ is an affine variety with an isolated singularity at 0 . With this observation in hand, for the remainder of this paper, we will work primarily with affine varieties, smooth away from 0, and comment on the Sasakian aspects of our work only where pertinent.

When defining test-configurations, we will need to consider degenerations of an affine variety into possibly non-reduced schemes, and we will need an algebro-geometric formulation of the notion of a Reeb vector field. Suppose that $Y \subset \mathbb{C}^{N}$ is an affine scheme, with a torus $T \subset A u t(Y)$. Let us write $\mathfrak{t}:=\operatorname{Lie}\left(T_{\mathbb{R}}\right)$ for the Lie algebra of the maximal compact sub-torus. Let $\mathcal{H}$ denote the global sections of the structure sheaf of $Y$, and write

$$
\mathcal{H}=\oplus_{\alpha \in \mathfrak{t}^{*}} \mathcal{H}_{\alpha}
$$

for the weight decomposition under the action of $T$.

Definition 2.3. A vector $\xi \in \mathfrak{t}$ is a Reeb vector field if for each non-empty weight space $\mathcal{H}_{\alpha}$, with $\alpha \neq 0$, we have $\alpha(\xi)>0$, i.e. $\xi$ acts with positive weights on the non-constant functions on $Y$. We will often identify the vector $\xi$ with the vector field it induces on $Y$. We define the Reeb cone to be

$$
\mathcal{C}_{R}:=\{\xi \in \mathfrak{t} \mid \xi \text { is a Reeb field }\} \subset \mathfrak{t} .
$$

Since $\mathcal{H}$ is finitely generated, $\mathcal{C}_{R}$ is a rational, convex, polyhedral cone, and for any $\xi \in \mathcal{C}_{R}$ there is an $\epsilon>0$ such that $\alpha(\xi) \geqslant \epsilon|\alpha|$ for all non-empty weight spaces. We say that $\xi$ is rational if there exists $\lambda \in \mathbb{R}_{>0}$ such that $\alpha(\lambda \xi) \in \mathbb{N}$ for every non-empty weight space. Otherwise, we say that $\xi$ is irrational.

Note that any homogeneous variety admits a Reeb field generated by the usual $\mathbb{C}^{*}$ action on $\mathbb{C}$. In analogy with this case, we shall call an affine scheme $Y$ with a holomorphic torus action admitting a Reeb vector field a polarized affine scheme. An affine scheme $Y$ may admit more than one Reeb field; choosing a Reeb vector field $\xi$ is analogous to fixing a polarization for a projective scheme. For the most part, we shall consider only polarized affine varieties. The next lemma shows that Reeb vector fields are always induced from Lie algebra actions on the ambient space, possibly after increasing the codimension of the embedding.

Lemma 2.1. Let $Y \subset \mathbb{C}^{N}$ be an affine scheme, and let $T$ be a torus acting holomorphically on $Y$. Then there exists an embedding $Y \hookrightarrow \mathbb{C}^{N^{\prime}}$ and a torus $T^{\prime} \subset G L\left(N^{\prime}, \mathbb{C}\right)$ such that the multiplicative action of $T^{\prime}$ on $\mathbb{C}^{N^{\prime}}$ induces the action of $T$ on $Y$.

Proof. Let $Y$ be cut out by the ideal $I \subset \mathbb{C}\left[x_{1}, \ldots, x_{N}\right]$, so that $Y=$ Spec $\mathcal{H}$ for $\mathcal{H}=\mathbb{C}\left[x_{1}, \ldots, x_{N}\right] / I$. The torus $T$ induces a decomposition

$$
\mathcal{H}=\oplus_{\alpha \in \mathfrak{t}^{*}} \mathcal{H}_{\alpha},
$$


and the images of $x_{1}, \ldots, x_{n}$ generate $\mathcal{H}$. In particular, there exists a finite set of homogenous generators $u_{1}, \ldots u_{N^{\prime}} \in \mathcal{H}$, with weights $\alpha_{1}, \ldots, \alpha_{N^{\prime}}$. Consider the map

$$
\begin{array}{cl}
\mathbb{C}\left[x_{1}, \ldots, x_{N^{\prime}}\right] & \longrightarrow \mathcal{H} \\
x_{i} & \longmapsto u_{i}
\end{array}
$$

Define an action of $T$ on $\mathbb{C}\left[x_{1}, \ldots, x_{N^{\prime}}\right]$, where $T$ acts on $x_{i}$ with weight $\alpha_{i}$. We get an exact sequence

$$
0 \longrightarrow I^{\prime} \longrightarrow \mathbb{C}\left[x_{1}, \ldots, x_{N^{\prime}}\right] \longrightarrow \mathcal{H} \longrightarrow 0
$$

which is equivariant with respect to the torus action. We obtain

$$
\text { Spec } \mathcal{H} \cong \operatorname{Spec} \frac{\mathbb{C}\left[x_{1}, \ldots, x_{N^{\prime}}\right]}{I^{\prime}} \hookrightarrow \operatorname{Spec} \mathbb{C}\left[x_{1}, \ldots, x_{N^{\prime}}\right],
$$

and hence an embedding $Y \hookrightarrow \mathbb{C}^{N^{\prime}}$. The action of $T$ on $Y$ is induced by the linear, diagonal action of $T$ on $\mathbb{C}^{N^{\prime}}$ as desired.

Because of this lemma, we are essentially dealing with affine schemes defined by ideals $I \subset \mathbb{C}\left[x_{1}, \ldots, x_{N}\right]$ for some $N$, which are homogeneous for the action of a torus $T \subset G L(N, \mathbb{C})$. We can even assume that the torus action is diagonal. A choice of an integral vector $\xi \in \mathfrak{t}$ then induces a grading on $\mathbb{C}\left[x_{1}, \ldots, x_{N}\right]$, which has positive weights when $\xi$ is a Reeb vector.

We will now relate our algebraic Reeb cone to the one defined differential geometrically in 25] (see also He-Sun [17, and the Sasaki Cone in [5]). Suppose that $Y \subset \mathbb{C}^{N}$ is an affine variety, smooth away from the origin, and $Y$ is defined by an ideal $I \subset \mathbb{C}\left[x_{1}, \ldots, x_{N}\right]$, homogeneous for the diagonal action of a torus $T$. We will also assume that $Y$ is not contained in a linear subspace.

Definition 2.4. A Kähler metric $\Omega$ on $Y$ is compatible with a Reeb vector field $\xi \in \mathfrak{t}$ if there exists a $\xi$-invariant function $r: Y \rightarrow \mathbb{R}_{>0}$ such that $\Omega=\frac{1}{2} i \partial \bar{\partial} r^{2}$ and $\xi=J\left(r \frac{\partial}{\partial r}\right)$, where $J$ denotes the restriction of the complex structure of $Y$.

Fixing a Reeb field, and a compatible metric is analogous to fixing an ample line bundle $L$, and choosing a metric in $c_{1}(L)$. To see this, let $Y$ be a polarized affine variety with $\operatorname{dim}_{\mathbb{C}} Y=n+1$, and let $\xi$ be a rational Reeb vector field. Let $\xi_{\mathbb{C}}$ be the complexification of $\xi$ and consider the holomorphic action induced by $\xi_{\mathbb{C}} \in \mathfrak{t}_{\mathbb{C}}$. Then $Y \backslash\{0\}$ is a principal $\mathbb{C}^{*}$ orbibundle over the orbifold $X=Y / \mathbb{C}^{*}$ corresponding to an ample orbi-line bundle $L \rightarrow X$. In particular, $Y \backslash\{0\}$ is the complement of the zero section in the total space of the orbi-line bundle $L^{-1}$. By the Kodaira-Bailey embedding theorem [4, the ampleness of $L$ is equivalent to the existence of a Hermitian metric $h$ on $L^{-1}$ such that $\omega=i \partial \bar{\partial} \log h$ is a metric on $X$. We define a function $r: Y \rightarrow \mathbb{R}_{>0}$ by $(z, \sigma) \rightarrow|\sigma|_{h(z)}$, for $\sigma$ in the fibre of $L^{-1}$ over $z \in X$. We get a metric on $Y$ by setting

$$
\Omega=i \partial \bar{\partial} r^{2}
$$


In particular, when $\xi$ is rational, $(Y, \xi)$ always admits a compatible Kähler metric.

Given a rational Reeb vector $\xi_{0}$, and compatible metric $\Omega_{0}$ on $Y$, the contact 1 -form $\eta_{0}$ is defined to be dual to $\xi_{0}$. The Reeb cone is defined in [17] to be

$$
\mathcal{C}_{R}^{\prime}=\left\{\xi \in \mathfrak{t} \mid \eta_{0}(\xi)>0 \text { on } Y \backslash\{0\}\right\} .
$$

Proposition 2.1. The cone $\mathcal{C}_{R}^{\prime}$ in (2) above coincides with the Reeb cone $\mathcal{C}_{R}$ that we defined in Definition [2.3.

Proof. We need to relate the condition that $\eta_{0}(\xi)>0$ with the weights of the circle action generated by $\xi$ on the ring of functions. As shown in [25], $H=\frac{1}{2} r^{2} \eta_{0}(\xi)$ is a Hamiltonian for the vector field $\xi$ with respect to $\Omega_{0}$. It follows that

$$
J \xi=-\nabla H,
$$

and moreover $H \rightarrow 0$ as we approach the cone point 0 .

Suppose first that $H$ is strictly positive, so $\xi$ cannot vanish anywhere. It follows that if we write $\phi_{t}: Y \rightarrow Y$ for the negative gradient flow of $H$, then

$$
\lim _{t \rightarrow \infty} \phi_{t}(p)=0
$$

for any $p \in Y$. Suppose that $f$ is a non-constant regular function on $Y$ (for instance a coordinate function on the ambient $\mathbb{C}^{N}$ ), on which $\xi$ acts with weight $\lambda$, and $p$ is a point such that $f(p) \neq 0$. Then $J \xi(f)=-\lambda f$, so

$$
\frac{d}{d t} f\left(\phi_{t}(p)\right)=-\lambda f\left(\phi_{t}(p)\right)
$$

Since $f(0)=0$, we must have $\lambda>0$. So if $\xi \in \mathcal{C}_{R}^{\prime}$, then $\xi \in \mathcal{C}_{R}$.

Conversely suppose that $H$ is negative somewhere. Since $H$ is homogeneous under $r \frac{\partial}{\partial r}$, we can then find points arbitrarily close to 0 , where $H$ is negative. For a suitable point $p$, the positive gradient flow $\phi_{t}$ of $H$ will satisfy $\phi_{t}(p) \rightarrow 0$ as $t \rightarrow \infty$. Then the same argument as above shows that if $f$ is a non-constant homogeneous function for $\xi$ which does not vanish at $p$, then the weight of $\xi$ on $f$ must be negative.

Corollary 2. If $\xi$ is an irrational Reeb vector field on $Y$ and $\Omega$ is a compatible Kähler metric, then there exists a sequence $\xi_{n} \mathfrak{t}$ of rational Reeb vector fields and compatible metrics $\Omega_{k}$ on $Y$, such that $\xi_{k} \rightarrow \xi$ in $\mathfrak{t}$, and the $\Omega_{k}$ converge to $\Omega$ smoothly on compact subsets of $Y$.

Proof. Since $\mathcal{C}_{R}$ is a rational convex polyhedral cone, we can approximate $\xi$ with a sequence of rational elements $\xi_{k} \in \mathcal{C}_{R}$. The construction of [17, Lemma 2.5] shows that there are compatible Kähler metrics $\Omega_{k}$, which will converge to $\Omega$ on compact subsets of $Y$. 
2.2. Orbifold K-stability. For a review of the basic properties of orbifolds with constant scalar curvature metrics in mind, see Ross-Thomas [31. Similarly to them, we will only be interested in polarized orbifolds, and as explained in 31] Remark 2.16., these can be viewed as global $\mathbb{C}^{*}$-quotients of affine schemes. More precisely, given a finitely generated graded ring

$$
R=\bigoplus_{k \geqslant 0} R_{k}
$$

over $\mathbb{C}$, the grading induces a $\mathbb{C}^{*}$-action on $\operatorname{Spec}(R)$. When $\operatorname{Spec}(R)$ is smooth, the corresponding orbifold is the quotient of $\operatorname{Spec}(R) \backslash\{0\}$ by this $\mathbb{C}^{*}$-action. More generally the quotient is a Deligne-Mumford stack. In our terminology below, the grading corresponds to a choice of rational Reeb field on the affine scheme $\operatorname{Spec}(R)$.

Differential geometrically the affine scheme $Y=\operatorname{Spec}(R)$, if smooth away from the origin, arises as the blowdown of the zero section in the total space of $L^{-1}$ for an orbifold $X$ with orbiample line bundle $L$. In Section 3 below we will express the Calabi functional on the orbifold $X$ in terms of a cone metric on $Y$. In the rest of this section we will review the work of [31] which gives a lower bound for the Calabi functional on the orbifold $X$ in terms of the Futaki invariants of test-configurations.

Roughly speaking a test-configuration for a polarized orbifold $(X, L)$ is a polarized, flat, $\mathbb{C}^{*}$-equivariant family over $\mathbb{C}$, whose generic fiber is $\left(X, L^{r}\right)$ for some $r>0$. In greatest generality the family should be allowed to be a Deligne-Mumford stack. For computations it is useful to reformulate this more algebraically. Let

$$
R=\bigoplus_{k \geqslant 0} H^{0}\left(X, L^{r}\right)
$$

be the homogeneous coordinate ring of $(X, L)$. Any set of homogeneous generators $f_{1}, \ldots, f_{k}$ of $R$ give rise to an embedding of $X \hookrightarrow \mathbb{P}$ into a weighted projective space. Assigning weights to the $f_{1}, \ldots, f_{k}$ induces a $\mathbb{C}^{*}$-action on the weighted projective space $\mathbb{P}$. Acting on $X \hookrightarrow \mathbb{P}$ we obtain a family $X_{t} \subset \mathbb{P}$ for $t \neq 0$. Taking the flat completion of this family across $t=0$ is a test-configuration $\chi$. The central fiber of this test-configuration is a polarized Deligne-Mumford stack $\left(X_{0}, L_{0}\right)$, with a $\mathbb{C}^{*}$-action. It is convenient to allow $L_{0}$ to be a $\mathbb{Q}$-line bundle, so that on the generic fiber we recover $L$ instead of a power of $L$. Let us write $d_{k}=\operatorname{dim} H^{0}\left(L_{0}^{k}\right)$ and let $w_{k}$ be the total weight of the $\mathbb{C}^{*}$-action on $H^{0}\left(L_{0}^{k}\right)$. As explained in [31], the Riemann-Roch theorem from Toën [39] implies that for large $k$ we have expansions

$$
\begin{aligned}
d_{k} & =a_{0} k^{n}+\left(a_{1}+\rho_{1}(k)\right) k^{n-1}+\ldots, \\
w_{k} & =b_{0} k^{n+1}+\left(b_{1}+\rho_{2}(k)\right) k^{n}+\ldots,
\end{aligned}
$$

where $\rho_{1}, \rho_{2}$ are periodic functions with average zero. The Futaki invariant of the test-configuration is then defined to be

$$
F u t(\chi)=\frac{a_{1}}{a_{0}} b_{0}-b_{1} \text {. }
$$


Writing $A_{k}$ for the infinitesimal generator of the $\mathbb{C}^{*}$-action on $H^{0}\left(L_{0}^{k}\right)$, there is also an expansion

$$
\operatorname{Tr}\left(A_{k}^{2}\right)=c_{0} k^{n+2}+O\left(k^{n+1}\right),
$$

and the norm of the test-configuration is defined by

$$
\|\chi\|^{2}=c_{0}-\frac{b_{0}^{2}}{a_{0}} .
$$

The main result that we need is the extension by Ross-Thomas 31 of Donaldson's lower bound for the Calabi functional [9], to orbifolds.

Theorem 2 (Donaldson, Ross-Thomas). Suppose that $(X, L)$ is a polarized orbifold of dimension $n$, and let $\omega \in c_{1}(L)$ be an orbifold metric. In addition suppose that $\chi$ is a test-configuration for $(X, L)$. Then

$$
\|\chi\| \cdot\left\|R_{\omega}-\hat{R}\right\|_{L^{2}(\omega)} \geqslant-c(n) F u t(\chi),
$$

where $R_{\omega}$ is the scalar curvature of $\omega$, and $\hat{R}$ is its average.

Although this result is not stated explicitly in [31], it follows easily from their proofs. In particular in their Theorem 6.6 the constant $C$ can be taken to be $\frac{1}{2}\left(\operatorname{vol} \sum_{i} c_{i}\right)^{1 / 2}$, while in the proof of Theorem 6.8 the constant $c$ equals $a_{0} \sum_{i} c_{i} k^{n+1}$ to highest order. Combining these, the last inequality in the proof of Theorem 6.8 gives the result we need.

\section{The Calabi Functional on a Polarized Affine Variety}

Let us suppose as in Section 2.1 that $Y \backslash\{0\}$ is the complement of the zero section in the total space of an orbi-line bundle $L^{-1}$ over $X$, and $h$ is a Hermitian metric on $L^{-1}$ such that $\omega=i \partial \bar{\partial} \log h$ is positive on $X$. Letting $r$ be the fiberwise norm, define the metric

$$
\Omega=i \partial \bar{\partial} r^{2}
$$

on $Y \backslash\{0\}$. We will compute the Calabi functional of $\omega$ in terms of the metric $\Omega$ on $Y$.

Fix local coordinates $(z, w)$ where $z \in X$ and $w$ is a local holomorphic section of $L^{-1}$ in a neighborhood of $p=\left(z_{0}, w_{0}\right)$, and assume that $d h=0$ at $p$. At $p$ we compute

$$
\Omega=i \partial \bar{\partial} h(z)|w|^{2}=r^{2} i \partial \bar{\partial} \log h+h(z) i \partial \bar{\partial}|w|^{2}=r^{2}\left(\pi^{*} \omega+\frac{i d w \wedge d \bar{w}}{|w|^{2}}\right) .
$$

Here $\pi: Y \rightarrow X$ is the natural projection map. It follows that the Ricci form and scalar curvature of $\Omega$ are given by

$$
\operatorname{Ric}(\Omega)=(n+1)\left(\pi^{*} \operatorname{Ric}(\omega)-\pi^{*} \omega\right), \quad R_{\Omega}=r^{-2}\left(\pi^{*} R_{\omega}-n\right) .
$$

On a fixed fibre, the cylinder metric $|w|^{-2}(d w \wedge d \bar{w})$ can also be written as $\frac{1}{r} d r \wedge d \theta$, where $d \theta$ is given by the $U(1)$ action on the fibres of $L^{-1}$. Hence, the volume form of $\Omega$ is

$$
\Omega^{n+1}=r^{2 n+1}\left(\pi^{*} \omega\right)^{n} \wedge d r \wedge d \theta
$$


Let $\left\{U_{i}, \Gamma_{i}\right\}, i=1, \ldots, n$ be a family of open sets $U_{i} \subset \mathbb{C}^{n}$ together with local uniformizing groups $\Gamma_{i}$, so that $U_{i} / \Gamma_{i} \cong V_{i} \subset X$ gives an open cover of $X$, and so that $L^{-1}$ is trivial on each $V_{i}$. Let $\phi_{i}$ be a partition of unity subordinate to the cover $V_{i}$. Note that the set $S:=\{r=1\} \subset Y$ is a smooth submanifold of $Y$, which is the total space of a principal $U(1)$ orbibundle over $X$. Thus, by Lemma 4.2 .8 of [7], we have that the local uniformizing groups inject into $U(1)$. In particular, the maps

$$
U(1) \times U_{i} \stackrel{\psi_{i}}{\longrightarrow} V_{i}
$$

are exactly $\left|\Gamma_{i}\right|$-to-one on the complement of the orbifold locus. Let $\hat{R}_{\omega}$ denote that average scalar curvature of $X$. We compute

$$
\begin{aligned}
\operatorname{Cal}_{X}(\omega)^{2}:=2 \pi \int_{X}\left(R_{\omega}-\hat{R}_{\omega}\right)^{2} \omega^{n} & =\sum_{i} \frac{2 \pi}{\left|\Gamma_{i}\right|} \int_{U_{i}} \phi_{i}\left(R_{\omega}-\hat{R}_{\omega}\right)^{2} \omega^{n} \\
& =\sum_{i} \frac{1}{\left|\Gamma_{i}\right|} \int_{U(1) \times U_{i}} \pi^{*} \phi_{i}\left(\pi^{*} R_{\omega}-\hat{R}_{\omega}\right)^{2} \pi^{*} \omega^{n} \wedge d \theta \\
& =\sum_{i=1}^{N} \int_{V_{i}} \pi^{*} \phi\left(\pi^{*} R_{\omega}-\hat{R}_{\omega}\right)^{2} \pi^{*} \omega^{n} \wedge d \theta \\
& =\int_{S}\left(\pi^{*} R_{\omega}-\hat{R}_{\omega}\right)^{2} \iota_{\frac{\partial}{\partial r}}\left(\Omega^{n+1}\right) .
\end{aligned}
$$

Let us write $\hat{R}_{\Omega}$ for the average of $R_{\Omega}$ when restricted to $S$. Then we have the relation

$$
\hat{R}_{\Omega}=\hat{R}_{\omega}-n
$$

Finally, we can compute

$$
\begin{aligned}
\int_{\{r \leq 1\} \subset Y}\left(r^{2} R_{\Omega}-\hat{R}_{\Omega}\right) \Omega^{n+1} & =\int_{0}^{1} \int_{S}\left(\pi^{*} R_{\omega}-\hat{R}_{\omega}\right)^{2} \iota_{\frac{\partial}{\partial r}}\left(\Omega^{n+1}\right) r^{2 n+1} d r \\
& =\frac{1}{2 n+2} \operatorname{Cal}_{X}(\omega)^{2} .
\end{aligned}
$$

Definition 3.1. Let $Y$ be an affine variety with isolated singular point at 0 , and Reeb field $\xi$ and let $\Omega$ be a Kähler metric on $Y$ compatible with $\xi$, with scalar curvature $R_{\Omega}$. Define

$$
\operatorname{Cal}_{Y}(\Omega):=\left(\int_{\{r \leq 1\}}\left(r^{2} R_{\Omega}-\hat{R}_{\Omega}\right)^{2} \Omega^{n+1}\right)^{1 / 2}
$$

where,

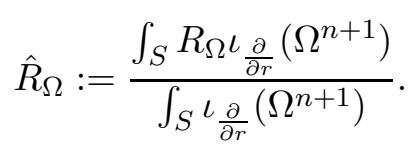


In order to relate this to the Sasakian setting, let $(S, g)$ be a Sasakian manifold. Observe that when $\xi$ is rational,

$$
\pi^{*} R_{\omega}=\frac{1}{4} R^{T}=\frac{1}{4}(R+2 n),
$$

where $R^{T}$ is the transverse scalar curvature of the Reeb foliation and $R$ is the scalar curvature of the Sasakian metric $g$. In this case, we have

$$
\begin{aligned}
\int_{S}\left(\pi^{*} R_{\omega}-\hat{R}_{\omega}\right)^{2} \iota_{\frac{\partial}{\partial r}}\left(\Omega^{n+1}\right) & =\frac{1}{16} \int_{S}\left(R^{T}-\hat{R}^{T}\right)^{2} \iota_{\frac{\partial}{\partial r}}\left(\Omega^{n+1}\right) \\
& =\frac{1}{16} \int_{S}(R-\hat{R})^{2} d \mu .
\end{aligned}
$$

Here $\hat{R}$ is the average scalar curvature of $(S, g)$. We note that this agrees, up to a constant, with the functional studied in [5]. We have shown that for rational Reeb fields we have the equality

$$
\operatorname{Cal}_{Y}(\Omega)=\frac{1}{4(2 n+2)^{1 / 2}} \operatorname{Cal}_{S}(g) .
$$

Since both sides of this equality depend continuously on the Reeb vector field, this also holds for irrational Reeb fields by approximation. We record this in the following proposition.

Proposition 3.1. Let $Y$ be an affine variety polarized by a rational Reeb field $\xi$. Then,

$$
\operatorname{Cal}_{Y}(\Omega)=\frac{1}{(2 n+2)^{1 / 2}} \operatorname{Cal}_{X}(\omega)
$$

Moreover, when $Y$ is the cone over a Sasakian manifold $(S, g)$, then

$$
\operatorname{Cal}_{Y}(\Omega)=\frac{1}{4(2 n+2)^{1 / 2}} \operatorname{Cal}_{S}(g)
$$

and this holds for any Reeb vector field.

Before proceeding, we make a few brief remarks about the scaling of the Calabi functional as a function of the Reeb field. More precisely, suppose that $Y$ is an affine cone with Reeb vector field $\xi$, and a compatible Kähler metric $\Omega=i \partial \bar{\partial} r^{2}$. Scaling the Reeb vector field by a factor $\lambda>0$, corresponds to changing $r$ by $r \mapsto r^{\lambda}$. This scaling yields a new metric $\Omega_{\lambda}=i \partial \bar{\partial} r^{2 \lambda}$. It is straight forward to check that under a deformation of this type we have

$$
\operatorname{Cal}_{Y}\left(\Omega_{\lambda}\right)=\lambda^{\frac{n-1}{2}} \operatorname{Cal}_{Y}(\Omega)
$$

\section{The Index Character and the Donaldson-Futaki Invariant}

The main difficulty in extending the definition of K-stability to irregular Sasakian manifolds, is the absence of a suitable Riemann-Roch formula when the Reeb field is irrational. When the Reeb field is rational, Ross-Thomas showed in 32 that the relevant coefficients are the non-periodic terms of the 
orbifold Riemann-Roch expansion (see Section 2.2). When the Reeb vector field is irrational, the Riemann-Roch expansion does not seem to exist, so we would like to define the relevant coefficients by approximating an irrational Reeb vector field $\xi$ by a sequence of rational ones $\xi_{k}$. The periodic terms in the expansions (3) corresponding to the $\xi_{k}$ become unmanageable as $k \rightarrow \infty$, so we need a different approach. The key observation is that the RiemannRoch coefficients are determined by the Hilbert series, or equivalently the index character introduced by Martelli-Sparks-Yau [25]. For the leading term (the volume), this was also used by [25].

In this section and the next we take $Y \subset \mathbb{C}^{N}$ to be an affine scheme of dimension $n+1$, defined by the ideal $I=\left(f_{1}, \ldots, f_{r}\right) \subset R=\mathbb{C}\left[x_{1}, \ldots, x_{N}\right]$. Let $T \subset G L(N, \mathbb{C})$ be a torus of dimension $s$ acting diagonally, holomorphically and effectively on $Y$. We make this assumption without loss of generality by Lemma 2.1. Denote by $\mathfrak{t}$ the Lie algebra of $T$ and let $\mathcal{H}=R / I$ be the ring of regular functions on $Y$. Since $T$ fixes $Y$, the ideal $I$ is homogeneous for the torus action. By Corollary 2 we may always assume that $T$ contains at least one rational Reeb vector field. Let

$$
\mathcal{H}=\oplus_{\alpha \in \mathfrak{t}^{*}} \mathcal{H}_{\alpha}
$$

be the weight space decomposition of $\mathcal{H}$.

Definition 4.1. In the above situation, we define the $T$-equivariant index character $F(\xi, t)$ for $\xi \in \mathcal{C}_{R}$ and $t \in \mathbb{C}$ with $\operatorname{Re}(t)>0$, by

$$
F(\xi, t):=\sum_{\alpha \in \mathfrak{t}^{*}} e^{-t \alpha(\xi)} \operatorname{dim} \mathcal{H}_{\alpha} .
$$

Lemma 4.1. The defining sum for $F(\xi, t)$ converges if $\xi$ is a Reeb vector field and $\operatorname{Re}(t)>0$.

Proof. The dimensions $\operatorname{dim} \mathcal{H}_{\alpha}$ are bounded by the corresponding dimensions for $\mathbb{C}^{N}$. As $\xi$ acts by positive weights, $\operatorname{dim} \mathcal{H}_{\alpha}<C|\alpha|^{N}$. Moreover, since $\xi$ is a Reeb vector field, there is a $c>0$ such that $\alpha(\xi)>c|\alpha|$ for all $\alpha$ with non-zero $\mathcal{H}_{\alpha}$. We obtain

$$
\sum_{\alpha \in \mathfrak{t}^{*}}\left|e^{-t \alpha(\xi)}\right| \operatorname{dim} \mathcal{H}_{\alpha} \leq C \sum_{\alpha \in \mathfrak{t}^{*}} e^{-c|\alpha| \operatorname{Re}(t)}|\alpha|^{N},
$$

which converges if $\operatorname{Re}(t)>0$.

Suppose that $\xi$ is rational, and it is minimal satisfying the condition that $\alpha(\xi)$ is integral for each $\alpha$ with non-zero weight space. Then as before we can think of $Y$ as the total space of a line bundle $L$ over the orbifold $X=Y / \mathbb{C}^{*}$, and

$$
H^{0}\left(X, L^{k}\right)=\bigoplus_{\alpha ; \alpha(\xi)=k} \mathcal{H}_{\alpha}
$$

By the orbifold Riemann-Roch theorem [18, 39], we have

$$
\operatorname{dim} H^{0}\left(X, L^{k}\right)=a_{0} k^{n}+\left(a_{1}+\rho\right) k^{n-1}+\cdots
$$


for some periodic function $\rho$ with average zero. In this case we have the following.

Proposition 4.1. The T-equivariant index character $F(\xi, t)$ as a function of $t$ has a meromorphic extension to a neighborhood of the origin, and it has Laurent expansion

$$
F(\xi, t)=\frac{a_{0} n !}{t^{n+1}}+\frac{a_{1}(n-1) !}{t^{n}}+O\left(t^{1-n}\right),
$$

near $t=0$.

Proof. By definition we have

$$
\begin{aligned}
F(\xi, t) & =\sum_{k=0}^{\infty} e^{-k t} \operatorname{dim} H^{0}\left(L^{k}\right) \\
& =\sum_{k=0}^{\infty} e^{-k t}\left(a_{0} k^{n}+\left(a_{1}+\rho\right) k^{n-1}+O\left(k^{n-2}\right)\right) .
\end{aligned}
$$

Note that

$$
\sum_{k} e^{-t k}=\frac{1}{1-e^{-t}}=\frac{1}{t}+f(t)
$$

where $f$ is analytic, so differentiating $n$ times with respect to $t$, we get

$$
\sum_{k} e^{-t k} k^{n}=\frac{n !}{t^{n+1}}+(-1)^{n} f^{(n)}(t) .
$$

Moreover, $G(t)=\sum_{k} \rho(k) e^{-t k}$ is analytic near $t=0$ since $\rho$ has average zero. Indeed if $d$ is the period of $\rho$ then we have

$$
\sum_{k}(\rho(k)+\rho(k+1)+\ldots+\rho(k+d-1)) e^{-t k}=0,
$$

and so

$$
G(t)+e^{t}(G(t)-\rho(0))+\ldots+e^{(d-1) t}\left(G(t)-\sum_{k=0}^{d-2} \rho(k) e^{-k t}\right)=0,
$$

and therefore

$$
G(t)=\frac{H(t)}{1+e^{t}+e^{2 t}+\ldots+e^{(d-1) t}}
$$

where $H(t)$ is analytic since it is a finite sum. It follows that $G(t)$ is also analytic near 0 , with poles at $t=\frac{2 \pi i k}{d}$ for non-zero integers $k \neq 0$. Finally it follows that $F(\xi, t)$ is meromorphic near $t=0$ with a pole at the origin, and we have

$$
F(\xi, t)=\frac{a_{0} n !}{t^{n+1}}+\frac{a_{1}(n-1) !}{t^{n}}+O\left(t^{1-n}\right) .
$$


In particular, we can read off the Riemann-Roch coefficients from the index character. The main advantage of this observation is that the index character is defined even when the Reeb vector field is irrational, and we can hope to use the asymptotics at $t=0$ to extract the coefficients $a_{0}, a_{1}$ which are needed for the definition of the Futaki invariant. In addition we will see that these coefficients vary smoothly as we vary $\xi$, so we will be able to use an approximation argument to prove our main result, Theorem 5. The difficutly of working more directly with the Riemann-Roch expansions on the quotient orbifolds is that as we vary the Reeb field, the orbifolds change, and the periodic terms in the expansions can become more and more unmanageable. The index character, on the other hand encodes the relevant coefficients in the Riemann-Roch expansions for all quotient orbifolds at the same time, so it becomes possible to study their variation as we vary $\xi$. In addition the index character is essentially the multivariate Hilbert series of a multigraded module, and thus it can be readily computed in examples.

The main observation is that fixing a Reeb field $\xi$ gives rise to a grading on $R=\mathbb{C}\left[x_{1}, \ldots, x_{N}\right]$, and on $R / I$, where $I$ cuts out the variety $Y$. Writing $H S_{R / I}(t)$ for the Hilbert series of the graded ring $R / I$, we have

$$
F(\xi, t)=H S_{R / I}\left(e^{-t}\right) .
$$

We will now assume that the embedding $Y \subset \mathbb{C}^{N}$ is obtained through an application of Lemma 2.1. The corresponding ideal $I \subset R$ is then homogeneous with respect to a multigrading on $R$.

More precisely, let $E:=\left\{e_{1}^{*}, \ldots, e_{s}^{*}\right\}$ be an integral basis of $\mathbb{R}^{s} \cong \mathfrak{t}^{*}$ and let $\alpha_{i}$ be the weight of the representation on the generator $x_{i}$ of $R$. Expressing the $\alpha_{i}$ in the basis $E$ yields an $s \times N$ matrix

$$
W=\left(\begin{array}{cccc}
\alpha_{1,1} & \alpha_{1,2} & \ldots & \alpha_{1, N} \\
\vdots & \vdots & \ddots & \vdots \\
\alpha_{s, 1} & \alpha_{s, 2} & \ldots & \alpha_{s, N}
\end{array}\right)
$$

with integer entries. Since, $R$ is graded by $W$, and $I$ is homogenous, it follows that $R / I$ is a $W$-graded $R$ module, generated in degree zero.

Definition 4.2. Let $s \geq 1$, let $R$ be graded by a matrix $W$ of rank $s$ in $M_{s, N}(\mathbb{Z})$, and let $\alpha_{1}, \ldots, \alpha_{s}$ be the rows of $W$. The grading on $R$ given by $W$ is of positive type if there exists $a_{1}, \ldots, a_{s} \in \mathbb{Z}$ such that all the entries of $a_{1} \alpha_{1}+\cdots+a_{s} \alpha_{s}$ are positive.

Lemma 4.2. If there exists a Reeb vector field in $\mathfrak{t}$, then the grading induced by $W$ is of positive type.

Proof. We first need to show that $W$ has rank $s$. Observe that if $v^{T} \cdot W=0$, then the action induced by $v$ is trivial. In particular, the action of $T$ is not effective. Secondly, by Corollary 2 we can assume that there is an integral Reeb field $\xi \in \mathfrak{t}$, given in terms of the dual basis $\left\{e_{1}, \ldots, e_{s}\right\}$ by a vector $\left(a_{1}, \ldots, a_{s}\right)$ with $a_{i} \in \mathbb{Z}$. The entries of $a_{1} \alpha_{1}+\ldots a_{s} \alpha_{s}$ are the weights of the 
action induced by $\xi$ on the generators $x_{1}, \ldots, x_{N}$. By definition of a Reeb field these are all positive.

We will now recall some results about multigradings and the multigraded Hilbert function.

Lemma 4.3 ([21], Proposition 4.1.19). Let $R=\mathbb{C}\left[x_{1}, \ldots, x_{N}\right]$ be graded by a matrix $W \in M a t_{m, N}(\mathbb{Z})$ of positive type, and let $M$ be a finitely generated graded $R$-module. Then,

(1) $R_{0}=\mathbb{C}$. That is, the degree zero elements in $R$ are precisely the constants.

(2) For every $d \in \mathbb{Z}^{m}$, we have $\operatorname{dim}_{\mathbb{C}}\left(M_{d}\right)<\infty$

The previous lemma indicates that the following definition makes sense;

Definition 4.3 (21], Definition 5.8.8, 5.8.11). Let $R$ be graded by a matrix $W \in \operatorname{Mat}_{m, N}(\mathbb{Z})$, and let $M$ be a finitely generated, graded $R$ module. Then the map

$$
\begin{aligned}
H F_{M, W}: \mathbb{Z}^{m} & \rightarrow \mathbb{Z} \\
\left(i_{1}, \ldots, i_{m}\right) & \mapsto \operatorname{dim}_{\mathbb{C}}\left(M_{i_{1}, \ldots, i_{m}}\right)
\end{aligned}
$$

for all $\left(i_{1}, \ldots, i_{m}\right) \in \mathbb{Z}^{m}$ is called the multigraded Hilbert function of $M$ with respect to the grading $W$. We may define the multivariate power Hilbert series of $M$ with respect to the grading $W$ by

$$
H S_{M, W}\left(z_{1}, \ldots z_{m}\right)=\sum_{\left(i_{1}, \ldots, i_{m}\right) \in \mathbb{Z}^{m}} H F_{M, W}\left(i_{1}, \ldots, i_{m}\right) z_{1}^{i_{1}} \cdots z_{m}^{i_{m}} \in \mathbb{Z}\left[\left[\mathbf{z}, \mathbf{z}^{-1}\right]\right]
$$

The following lemma provides a convenient characterization of multivariate Hilbert series under changes in the grading.

Lemma 4.4 ([21], Proposition 5.8.24). Let $W \in \operatorname{Mat}_{m, N}(\mathbb{Z})$, and $A=$ $\left(a_{i j}\right) \in \operatorname{Mat}_{l, m}(\mathbb{Z})$ be two matrices such that the gradings on $R=\mathbb{C}\left[z_{0}, \ldots, z_{N}\right]$ given by $W$ and $A \cdot W$ are both of positive type. Let $M$ be a finitely generated $R$-module which is graded with respect to the grading given by $W$. Then the Hilbert series of $M$ with respect to the grading given by $A \cdot W$ is given by

$$
H S_{M, A \cdot W}\left(z_{1}, \ldots, z_{l}\right)=H S_{M, W}\left(z_{1}^{a_{11}} \cdots z_{l}^{a_{l 1}}, \ldots, z_{1}^{a_{1 m}} \cdots z_{l}^{a_{l m}}\right)
$$

If $\mathrm{R}$ is graded by $W=\left(w_{i j}\right) \in \operatorname{Mat}_{m, n}(\mathbb{Z})$ of positive type, and $\xi$ is a Reeb field, then the grading induced by $\xi^{T} \cdot W$ is clearly of positive type, and so the above lemma describes the relation between the multigraded Hilbert series and the index character. The next proposition describes the general shape of multivariable Hilbert series.

Proposition 4.2 ([21], Corollary 5.8.19). Let $R$ be graded by $W \in M a t_{m, N}(\mathbb{Z})$, a matrix of positive type. Let $M$ be a finitely generated, graded $R$-module, and $\left(m_{1}, \ldots, m_{r}\right)$ be a tuple of non-zero homogeneous elements of $M$ which 
form a minimal system of generators. For $i=1, \ldots, r$, let $d_{i}=\operatorname{deg}_{W}\left(m_{i}\right)$. Then the multivariate Hilbert series of $M$ has the following form;

$$
H S_{M, W}\left(z_{1}, \ldots z_{m}\right)=\frac{z_{1}^{\alpha_{1}} \cdots z_{m}^{\alpha_{m}} \cdot H N\left(z_{1}, \ldots, z_{m}\right)}{\prod_{j=1}^{N}\left(1-z_{1}^{w_{1 j}} \cdots z_{m}^{w_{m j}}\right)}
$$

where $\left(\alpha_{1}, \ldots, \alpha_{m}\right)$ is the component wise minimum of $\left\{d_{i}\right\}$, and $H N_{M}\left(z_{1}, \ldots, z_{m}\right)$ is a polynomial in $\mathbb{Z}\left[z_{1}, \ldots, z_{m}\right]$.

We can now translate this result to the language of index characters.

Theorem 3. Let $Y \subset \mathbb{C}^{N}$ be an affine scheme of dimension $n+1$, and suppose that $T \subset G L(N, \mathbb{C})$ is a torus acting effectively, diagonally and holomorphically on $Y$. Let $\mathfrak{t}$ be the Lie algebra of $T$, and $\mathcal{C}_{R} \subset \mathfrak{t}$ be the Reeb cone. For fixed $\xi \in \mathcal{C}_{R}$ the index character $F(\xi, t)$ has a meromorphic extension to $\mathbb{C}$ with poles along the imaginary axis. Near $t=0$ it has a Laurent series

$$
F(\xi, t)=\frac{a_{0}(\xi) n !}{t^{n+1}}+\frac{a_{1}(\xi)(n-1) !}{t^{n}}+\ldots,
$$

where $a_{i}(\xi)$ depend smoothly on $\xi \in \mathcal{C}_{R}$, and $a_{0}(\xi)>0$.

Proof. As above, with a basis of $\mathfrak{t} \cong \mathbb{R}^{s}$ fixed, write $\xi=\left(\xi_{1}, \ldots, \xi_{s}\right)$ for an element of $\mathfrak{t}$. By Proposition 4.2, the Hilbert series of the grading induced by $W$ is given by

$$
H S_{W}\left(e^{-t_{1}}, \ldots, e^{-t_{s}}\right)=\frac{e^{-t_{1} \alpha_{1}} \cdots e^{-t_{s} \alpha_{s}} \cdot H N\left(e^{-t_{1}}, \ldots, e^{-t_{s}}\right)}{\prod_{j=1}^{N}\left(1-e^{-t_{1} w_{1 j}} \cdots e^{-t_{s} w_{s j}}\right)}
$$

where $\alpha_{i} \geq 0$ for every $i$. By Lemma 4.4, we obtain

$$
F(\xi, t)=H S_{\xi^{T} \cdot W}\left(e^{-t}\right)=\frac{e^{-t\left(\xi_{1} \alpha_{1}+\cdots+\xi_{s} \alpha_{s}\right)} \cdot H N\left(e^{-t \xi_{1}}, \ldots, e^{-t \xi_{s}}\right)}{\prod_{j=1}^{N}\left(1-e^{-t\left(\xi_{1} w_{1 j}+\cdots+\xi_{s} w_{s j}\right)}\right)}
$$

From this formula it follows that $F(\xi, t)$ is a meromorphic function with coefficients depending smoothly on the Reeb field. More precisely for fixed $\xi \in \mathcal{C}_{R}$ there are no poles other than the origin in the ball where

$$
|t|<\frac{2 \pi}{\max _{j}\left\{\xi_{1} w_{1 j}+\ldots+\xi_{s} w_{s j}\right\}},
$$

so we can compute the coefficients of the Laurent series using the Cauchy integral formula on a small circle around the origin. As long as $\xi$ varies in a bounded subset of $\mathcal{C}_{R}$, we can use the same circle around the origin, and the coefficients will vary smoothly with $\xi$. It follows also that the order of the pole at $t=0$ is determined by the order of the pole for rational $\xi$, which is $n+1$ by Proposition 4.1. Note that the coefficients blow up at the boundary of the Reeb cone, since as $\xi$ approaches the boundary, there will be a $j$ such that $\xi_{1} w_{1 j}+\ldots+\xi_{s} w_{s j} \rightarrow 0$.

In some special cases, we can recover this result by computing the index character explicitly. For example, we have 
Proposition 4.3. Let $Y$ be a complete intersection, determined by the regular sequence $f_{1}=\cdots=f_{k}=0$. Let $\alpha_{i}$ be the weight of the generators $x_{i}$, and let $\beta_{j}$ be the weight of $f_{j}$. Then we have

$$
F(\xi, t)=\frac{\prod_{j=1}^{k}\left(1-e^{-t \beta_{j}(\xi)}\right)}{\prod_{i=0}^{N}\left(1-e^{-t \alpha_{i}(\xi)}\right)}
$$

Proof. Use the degree shifted Koszul complex resolution of $R / I$, and compute the Hilbert series.

In order to define the Futaki invariant, we need equivariant versions of the index character, taking into account an extra $\mathbb{C}^{*}$-action.

Definition 4.4. In the situation of Theorem 3 with $\xi \in \mathcal{C}_{R}$, suppose $\eta \in \mathfrak{t}$, and define the weight characters

$$
\begin{aligned}
C_{\eta}(\xi, t) & =\sum_{\alpha \in \mathfrak{t}^{*}} e^{-t \alpha(\xi)} \alpha(\eta), \\
C_{\eta^{2}}(\xi, t) & =\sum_{\alpha \in \mathfrak{t}^{*}} e^{-t \alpha(\xi)}(\alpha(\eta))^{2} .
\end{aligned}
$$

The convergence of these weight character follows from the arguments in Lemma 4.1. As before, when $\xi$ is rational, we obtain a line bundle $L$ over the orbifold $X=Y / \mathbb{C}^{*}$ with a $\mathbb{C}^{*}$-action on $L$ generated by $\eta$. Adapting the computations preceeding Proposition 4.1 proves the following.

Proposition 4.4. In the situation of Theorem [3, with $\xi$ rational, write $A_{k}$ for the infinitesimal action of $\eta$ on $H^{0}\left(X, L^{k}\right)$, and define $b_{0}, b_{1}, c_{0}$ by the expansions

$$
\begin{aligned}
& \operatorname{Tr}\left(A_{k}\right)=b_{0} k^{n+1}+\left(b_{1}+\rho\right) k^{n}+O\left(k^{n-1}\right), \\
& \operatorname{Tr}\left(A_{k}^{2}\right)=c_{0} k^{n+2}+O\left(k^{n+1}\right),
\end{aligned}
$$

where $\rho$ is a periodic function with average zero, and $c_{0} \geq 0$. Then the weight characters have the asymptotic expansions

$$
\begin{gathered}
C_{\eta}(\xi, t)=\frac{b_{0}(n+1) !}{t^{n+2}}+\frac{b_{1} n !}{t^{n+1}}+O\left(t^{-n}\right), \\
C_{\eta^{2}}(\xi, t)=\frac{c_{0}(n+2) !}{t^{n+3}}+O\left(t^{-n-2}\right) .
\end{gathered}
$$

We remark that the inequality $c_{0} \geq 0$ follows from equation (2.20) in [32], orThe results of Theorem 3 can also be extended quite easily.

Theorem 4. In the situation of Theorem 3, with $\eta \in \mathfrak{t}$, the weight characters admit meromorphic expansions to a small neighbourhood of $0 \in \mathbb{C}$ of the form

$$
\begin{aligned}
C_{\eta}(\xi, t) & =\frac{b_{0}(\xi)(n+1) !}{t^{n+2}}+\frac{b_{1}(\xi) n !}{t^{n+1}}+O\left(t^{-n}\right) \\
C_{\eta^{2}}(\xi, t) & =\frac{c_{0}(\xi)(n+2) !}{t^{n+3}}+O\left(t^{-n-2}\right),
\end{aligned}
$$


where $b_{0}, b_{1}, c_{0}$ depend smoothly on $\xi \in \mathcal{C}_{R}$. Moreover, we have

$$
\begin{aligned}
& b_{i}(\xi)=\frac{-1}{(n+1-i)} D_{\eta} a_{i}(\xi) \quad \text { for } i=0,1 \\
& c_{0}(\xi)=\frac{1}{(n+2)(n+1)} D_{\eta}^{2} a_{0}(\xi),
\end{aligned}
$$

where $D_{\eta}$ denotes the directional derivative along $\eta$ in $\mathbb{R}^{s} \cong \mathfrak{t}$.

Proof. We define

$$
G(\xi, s, t)=\sum_{\alpha \in \mathfrak{t}^{*}} e^{-t \alpha(\xi-s \eta)} \operatorname{dim} H_{\alpha}
$$

For $s$ sufficiently small, $\xi-s \eta$ is a Reeb vector field and so the defining sum for $G(\xi, s, t)$ converges uniformly for $t>0$, and we have $G(\xi, s, t)=$ $F(\xi-s \eta, t)$. It is clear that

$t C_{\eta}(\xi, t)=\left.\frac{\partial}{\partial s} G(\xi, s, t)\right|_{s=0}=\left.\frac{\partial}{\partial s}\left(\frac{a_{0}(\xi-s \eta) n !}{t^{n+1}}+\frac{a_{1}(\xi-s \eta)(n-1) !}{t^{n}}+\cdots\right)\right|_{s=0}$.

By Theorem 3 the coefficients $a_{0}, a_{1}, \ldots$ depend smoothly on the Reeb field and so we can differentiate term by term to obtain

$$
C_{\eta}(\xi, t)=\frac{b_{0}(\xi)(n+1) !}{t^{n+2}}+\frac{b_{1}(\xi) n !}{t^{n+1}}+\cdots
$$

where, for example, $b_{0}(\xi)=\frac{-1}{n+1} D_{\eta} a_{0}(\xi)$ and $D_{\eta}$ denotes the directional derivative along $\eta$. The argument for $C_{\eta^{2}}$ is identical.

\section{Test Configurations for Polarized Affine Varieties}

Our first task is to define a test configuration for an affine variety $Y$ polarized by a Reeb field $\xi$. Recall that we can assume that $Y \subset \mathbb{C}^{N}$ is invariant under the linear action of a torus $T$ and the Reeb field $\xi$ is in the Lie algebra $\mathfrak{t}$ of the maximal compact subtorus. Let $\mathcal{H}$ be the coordinate ring of $Y$.

Definition 5.1. A T-equivariant test-configuration for $Y$ consists of the following data.

(1) A set of $T$-homogeneous elements $\left\{f_{1}, \ldots, f_{k}\right\} \in \mathcal{H}$, which generate $\mathcal{H}$ in sufficiently high degrees.

(2) Integers $w_{i}$ for $i=1, \ldots, k$.

This corresponds to the usual, more geometric definition of test-configurations. Namely we can embed $Y$ into $\mathbb{C}^{k}$ using the functions $\left\{f_{1}, \ldots, f_{k}\right\}$, and then act on $\mathbb{C}^{k}$ by the $\mathbb{C}^{*}$-action with weights $w_{i}$. Taking the flat limit across 0 of the $\mathbb{C}^{*}$-orbit of $Y$ we obtain a flat family of affine schemes over $\mathbb{C}$. It is in this form that we will construct our test configurations in Section 6 . The central fiber $Y_{0}$ still has an action of $T$ as well as a new $\mathbb{C}^{*}$-action commuting with $T$ (if we have a product configuration, then this new $\mathbb{C}^{*}$ is actually a subgroup of $T$ ). Note that when $\xi$ is rational, then we can take 
$T$ to be the 1-dimensional torus generated by $\xi$, and a test-configuration for $Y$ is the same as a test-configuration for the quotient orbifold as we defined it in Section 2.2.

It is important to note that as a $T$-representation, the ring of functions on the central fiber $Y_{0}$ is isomorphic to $\mathcal{H}$, it is only the multiplicative structure that changes. In particular if $\xi \in \mathfrak{t}$ is a Reeb field on $Y$, then it is also a Reeb field on $Y_{0}$. We can therefore apply our results on the index character to $Y_{0}$. By Theorem 3 , the index character expands asymptotically as

$$
F(\xi, t)=\frac{a_{0}(\xi) n !}{t^{n+1}}+\frac{a_{1}(\xi)(n-1) !}{t^{n}}+O\left(t^{1-n}\right)
$$

where $a_{0}, a_{1}: \mathcal{C}_{R} \rightarrow \mathbb{R}$ are smooth functions. Moreover, $Y$ inherits an extra $\mathbb{C}^{*}$ action generated by $\eta \in \mathfrak{t}^{\prime}=\operatorname{Lie}\left(T_{\mathbb{R}}^{\prime}\right)$ for some torus $T^{\prime} \subset G L(N, \mathbb{C})$ with $T \subset T^{\prime}$. By Theorem 4 , the weight characters expand as

$$
\begin{aligned}
C_{\eta}(\xi, t) & =\frac{b_{0}(\xi)(n+1) !}{t^{n+2}}+\frac{b_{1}(\xi) n !}{t^{n+1}}+O\left(t^{-n}\right) \\
C_{\eta^{2}}(\xi, t) & =\frac{c_{0}(\xi)(n+2) !}{t^{n+3}}+O\left(t^{2-n}\right)
\end{aligned}
$$

where $b_{0}, b_{1}, c_{0}: \mathcal{C}_{R} \rightarrow \mathbb{R}$ are smooth functions, and $c_{0} \geq 0$.

Definition 5.2. In the above situation, we define the Donaldson-Futaki invariant of the test configuration, with respect to the Reeb field $\xi$, by

$$
F u t\left(Y_{0}, \xi, \eta\right):=\frac{a_{1}(\xi)}{a_{0}(\xi)} b_{0}(\xi)-b_{1}(\xi)=\frac{a_{0}(\xi)}{n} D_{\eta}\left(\frac{a_{1}}{a_{0}}\right)(\xi)+\frac{a_{1}(\xi) D_{\eta} a_{0}(\xi)}{n(n+1) a_{0}(\xi)},
$$

where the second equality follows from Theorem 4. We also define the norm of $\eta$, with respect to the Reeb field $\xi$ by

$$
\|\eta\|_{\xi}^{2}=c_{0}(\xi)-\frac{b_{0}(\xi)^{2}}{a_{0}(\xi)}
$$

Propositions 4.1 and 4.4 show that the above definition of the DonaldsonFutaki invariant extends Ross-Thomas's orbifold Donaldson-Futaki invariant to irrational Reeb vector fields.

Definition 5.3. We say that $(Y, \xi)$ is K-semistable if, for every torus $T \ni \xi$, and every $T$-equivariant test configuration with central fibre $Y_{0}$, we have

$$
\operatorname{Fut}\left(Y_{0}, \xi, \eta\right) \geq 0
$$

where $\eta \in T^{\prime}$ is the induced $\mathbb{C}^{*}$ action on the central fibre.

K-stability could also be defined along similar lines. Since there is usually a positive dimensional torus of automorphisms, one natural way would be to use the notion of relative stability following [37. This would also allow us to consider the analogs of extremal metrics (called canonical Sasakian metrics in [5]). Since we do not use these notions in this paper, we will not define them. We are now in a position to prove our main theorem; 
Theorem 5. Let $(Y, \xi)$ be a polarized affine variety of dimension $n+1$ with a torus of automorphisms T, containing the Reeb field. Suppose that we have a T-equivariant test-configuration for $Y$ and let $Y_{0}$ be the central fibre with induced $\mathbb{C}^{*}$-action $\eta$. For any Kähler metric $\Omega$ on $Y$ compatible with $\xi$,

$$
\|\eta\|_{\xi} \cdot \operatorname{Cal}_{Y}(\Omega) \geq-c(n)(\xi) F u t\left(Y_{0}, \xi, \eta\right),
$$

where $c(n)$ is a strictly positive constant depending only on $n$.

Proof. When $\xi$ is rational and minimal satisfying the condition that $\alpha(\xi)$ is integral for each $\alpha \in \mathfrak{t}^{*}$ with non-empty weight space, this theorem is just a restatement of the results of Donaldson [9] and Ross-Thomas [32. However, from the definitions of $a_{i}, b_{i}, c_{0}$ for $i=0,1$, and the scaling of the Calabi functional in equation (15), the inequality is invariant under scaling the Reeb field. In particular, it holds for all rational Reeb vector fields.

Assume that $\xi$ is irrational. According to Corollary 2 we can approximate $\xi$ with a sequence of rational Reeb fields $\xi_{k} \in \mathfrak{t}$, and find corresponding compatible Kähler metrics $\Omega_{k}$, which converge to $\Omega$ smoothly on compact sets. For the rational $\xi_{k}$ we already know that

$$
\|\eta\|_{\xi_{k}} \cdot \operatorname{Cal} l_{Y}\left(\Omega_{k}\right) \geq-c(n) F u t\left(Y_{0}, \xi_{k}, \eta\right) .
$$

All the terms in this inequality depend smoothly on the Reeb vector field by Theorems 3 and 4 , and since $\Omega_{k} \rightarrow \Omega$ smoothly on compact sets. We can therefore take a limit as $k \rightarrow \infty$ to obtain the inequality for the irrational Reeb field $\xi$.

Corollary 1, stated in the introduction, follows immediately from Theorem 5, since $c(n)>0$.

\section{Applications and Examples}

As an application of our techniques, we will show that the the volume minimization results of [25] and the Lichnerowicz obstruction of [16] can be obtained directly from K-stability considerations as obstructions to existence of Sasaki-Einstein metrics. More precisely, we will show that for Calabi-Yau

cones with isolated Gorenstein singularities, and a torus action inducing a Reeb vector field, K-stability for product test configurations implies the volume minimization results of [25]. Martelli, Sparks and Yau noticed that when the Reeb field minimizing the volume functional was rational, the Futaki invariant on the quotient orbifold vanished. Secondly, we will apply the Rees deformation to interpret the Lichnerowicz obstruction of [16] in terms of K-stability. For rational Reeb vector fields, the Lichnerowicz obstruction was shown to imply the slope instability, and hence K-instability, of the quotient orbifold in [32]. Our results recover this theorem, and extend it to the setting of irrational Reeb fields.

Let $Y$ be an affine, Calabi-Yau variety with an isolated singularity at 0 , and a torus $T$, acting holomorphically, and effectively on $Y$, admitting a Reeb vector field $\xi \in \mathfrak{t}$. We suppose that $0 \in Y$ is a Gorenstein singularity, 
by which we mean that the canonical bundle is trivial on $X:=Y-\{0\}$. According to section 2.7 of [25], we fix a non-vanishing section $\Theta \in H^{0}\left(X, K_{X}\right)$ which is homogeneous of degree $n+1$ for the action of the Reeb field. More precisely, we fix a cross-section $\Sigma \subset \mathcal{C}_{R}$ so that for each $\xi \in \Sigma$, we have $\mathcal{L}_{\xi} \Theta=i(n+1) \Theta$. According to [25], $\Sigma$ is a compact, convex polytope. By the computations in Section 3.1 of 25$]$

$$
\int_{S} R\left(g_{S}\right) d \mu=2 n(2 n+1) \operatorname{Vol}(S) .
$$

Assuming for the moment that $\xi \in \Sigma$ is rational, the orbifold Riemann-Roch theorem implies that

$$
a_{1}(\xi)=\frac{1}{2} \int_{X} R_{\omega} \frac{\omega^{n}}{n !}=\frac{1}{16 \pi} \int_{S} R\left(g_{S}\right) d \mu+\frac{2 n}{16 \pi} \operatorname{Vol}(S) .
$$

This follows from a computation similar to the computation in section 4 for the Calabi functional, and the relation between the complex transverse scalar curvature of the Reeb foliation and the real scalar curvature of the Sasakian metric given by equation (44). Moreover, we have

$$
a_{0}(\xi)=\frac{1}{2 \pi} \operatorname{Vol}(S),
$$

which follows easily by a similar argument. Since both of these identities are continuous in the Reeb field, they extend from the rational Reeb fields to all of $\Sigma$. Thus, for $\xi \in \Sigma$ we have

$$
a_{1}(\xi)=\frac{n(n+1)}{2} a_{0}(\xi) .
$$

Consider now a product test configuration $Y \times \mathbb{C}$, with a $\mathbb{C}^{*}$ action generated by $\eta \in \mathfrak{t}$. We assume additionally that $\eta$ is tangent $\Sigma$. Applying equation (10), the Donaldson-Futaki invariant is given by

$$
\operatorname{Fut}(Y, \xi, \eta)=\frac{1}{2} D_{\eta} a_{0}(\xi) .
$$

Since we could replace $\eta$ with $-\eta$, it follows from Theorem 5 that If $\xi$ is the Reeb vector field of a Sasaki-Einstein metric, then we must have

$$
D_{\eta} a_{0}(\xi)=0
$$

for every rational $\eta$, and hence $\xi$ must be a critical point of the volume functional. Moreover, it was shown in 25] that the volume functional of a Sasakian manifold is strictly convex when restricted to $\Sigma$, so a critical point is necessarily a minimum. In particular, we have

Theorem 6. Let $(Y, \Theta)$ be an isolated Gorenstein singularity with link L, and Reeb vector field $\xi$ satisfying $\mathcal{L}_{\xi} \Theta=i(n+1) \Theta$. If $\xi$ does not minimize the volume functional of the link $L$, then $(Y, \xi)$ is K-unstable.

From the argument it is clear that more generally in any family of Reeb fields $\xi$ for which the ratio $a_{1} / a_{0}$ is constant, a K-semistable Reeb field must 
be a critical point of the volume $a_{0}$. In the case of Gorenstein singularities we obtain the following corollary, which was first pointed out in [25].

Corollary 3. Let $(Y, \Theta)$ be an isolated Gorenstein singularity with link L, and Reeb vector field $\xi$ satisfying $\mathcal{L}_{\xi} \Theta=i(n+1) \Theta$. If $\xi$ does not minimize the volume functional of the link $L$, then $(Y, \xi)$ does not admit a compatible Kähler metric with constant scalar curvature. In particular, the link $L$ with Reeb field $\xi$ does not admit a Sasaki-Einstein metric.

Next, we aim to show how the Lichnerowicz obstruction of Gauntlett, Martelli, Sparks and Yau [16] can be interpreted in terms of K-stability by computing explicitly the Donaldson-Futaki invariant of a test configuration arising from the Rees algebra for a principal ideal. These test configurations, which we call the Rees deformation, are a simplified version of the deformation to the normal cone test configurations studied by Ross-Thomas [32, 31]. Let $R=\mathbb{C}\left[x_{1}, \ldots, x_{N}\right] /\left(f_{1}, \ldots, f_{d}\right)$, and $Y=$ Spec $R$ be an affine variety with an effective, holomorphic action action of a torus $T$, and let $V \subset Y$ be an invariant subscheme, corresponding to a homogenous ideal $I \subset R$. Suppose that $\xi \in \mathfrak{t}$ is a Reeb vector field. We consider the Rees algebra of $R$ with respect to $I$, given by

$$
\mathcal{R}=\mathcal{R}(R, I):=\bigoplus_{n \in \mathbb{Z}} t^{-n} I^{n}=R\left[t, t^{-1} I\right] \subset R\left[t, t^{-1}\right]
$$

where $I^{n}:=R$ for $n \leq 0$. For ease of notation we set $\mathcal{Y}=\operatorname{Spec} \mathcal{R}$. Note that $\mathcal{Y}$ admits a $\mathbb{C}^{*}$ action induced by $\lambda \cdot t=\lambda^{-1} t$ for $\lambda \in \mathbb{C}^{*}$. The canonical inclusion $\mathbb{C}[t] \hookrightarrow \mathcal{R}$ gives a map $\pi: \mathcal{Y} \rightarrow \mathbb{C}$, and this map is clearly $\mathbb{C}^{*}$ equivariant with respect to the above action. The scheme $\mathcal{Y}$ carries a natural action of $T$ by acting on the $t$-graded components, and hence commuting with the $\mathbb{C}^{*}$ action. For $\alpha \in \mathbb{C}-\{0\}$, the fibre $\pi^{-1}(\alpha) \cong Y$, as $\mathcal{R} /(T-\alpha) \mathcal{R} \cong$ $R$, and so the generic fibre is isomorphic to $Y$. The $T$ action on $\mathcal{Y}$ clearly preserves the fibres, and restricts to the action of $T$ on $Y$ away from the central fibre. Moreover, we have

$$
\mathcal{Y}_{0}:=\pi^{-1}(0)=\operatorname{Spec} \bigoplus_{n \geq 0} I^{n} / I^{n+1},
$$

and so the central fibre is precisely the normal cone of $V$ in $Y$. The $\mathbb{C}^{*}$ action on the central fibre is determined by the grading giving $I^{n} / I^{n+1}$ degree $n$. Moreover, if $\xi \in \mathfrak{t}$ is the Reeb field, then $\xi$ induces a Reeb field on $\mathcal{Y}_{0}$. To see this, observe that if $\xi$ induces a positive grading on $R$, and $I \subset R$ is homogeneous, then $\xi$ also induces a positive grading on

$$
R / I \oplus I / I^{2} \oplus \cdots \oplus I^{n} / I^{n+1} \oplus \cdots
$$

Finally, it is well known that $\mathcal{R}(R, I)$ is flat over $\mathbb{C}[t]$; see for instance [12].

In order to obtain the Lichnerowicz obstruction, we consider the simplest family Rees deformations; namely, those obtained from principal ideals. Fix a holomorphic function $f: Y \rightarrow \mathbb{C}$, which is homogeneous for the torus action. We denote by $\alpha \in \mathfrak{t}^{*}$ the weight of $f$ under $T$. Consider the ideal $I=$ 
$(f) \subset R$, and the test configuration given by the Rees algebra $\mathcal{R}(R, I)$. The central fibre, which we denote by $Y_{0}$, of this test configuration is determined by the ring

$$
\bigoplus_{n \geq 0} I^{n} / I^{n+1} \cong R / I \otimes_{\mathbb{C}} \mathbb{C}[w] .
$$

The grading on the latter ring is induced by the torus $T$ on the first factor. The torus action on the second factor is by weight $\alpha$ on $w$. Finally, the induced $\mathbb{C}^{*}$ action, denoted $\eta$, on the central fibre is trivial on $R / I$, and acts with weight 1 on $w$. We can compute the Donaldson-Futaki invariant of this test configuration entirely in terms of the weight of the torus action on $f$, and the Hilbert series of $R$. First, we observe that if $H_{R}\left(z_{0}, \ldots, z_{s}\right)$ is the Hilbert series of $R$ with multigrading induced by $T$, and $\alpha_{0}, \ldots, \alpha_{s}$ denote the weight of $f$ under multigrading, then

$$
H_{R / I}\left(z_{0}, \ldots, z_{s}\right)=\left(1-z_{0}^{\alpha_{0}} \cdots z_{s}^{\alpha_{s}}\right) H_{R}\left(z_{0}, \ldots, z_{s}\right)
$$

is the Hilbert series of $R / I$. This follows immediately from the degree shifted exact sequence

$$
0 \longrightarrow R^{\left[\alpha_{0}, \ldots, \alpha_{s}\right]} \stackrel{f}{\longrightarrow} R \longrightarrow R / I \longrightarrow 0 .
$$

Since the Hilbert series is multiplicative on tensor products, we have

$$
H_{R / I \otimes_{R} \mathbb{C}[w]}\left(z_{0}, \ldots, z_{s}, \tilde{z}\right)=\frac{\left(1-z_{0}^{\alpha_{0}} \cdots z_{s}^{\alpha_{s}}\right)}{\left(1-z_{0}^{\alpha_{0}} \cdots z_{s}^{\alpha_{s}} \tilde{z}\right)} H_{R}\left(z_{0}, \ldots, z_{s}\right) .
$$

Suppose that the index character of $Y$ with Reeb field $\xi \in \mathfrak{t}$ expands as

$$
F(\xi, t)=\frac{a_{0}(\xi) n !}{t^{n+1}}+\frac{a_{1}(\xi)(n-1) !}{t^{n}}+O\left(t^{1-n}\right) .
$$

then one easily obtains that the index character of the central fibre is given by

$$
\begin{aligned}
F(\xi-s \eta, t) & =\frac{1-e^{-t \alpha(\xi)}}{\left(1-e^{-t(\alpha(\xi)-s)}\right)}\left(\frac{a_{0}(\xi) n !}{t^{n+1}}+\frac{a_{1}(\xi)(n-1) !}{t^{n}}+O\left(t^{1-n}\right)\right) \\
& =\frac{a_{0}(\xi) \alpha(\xi) n !}{(\alpha(\xi)-s) t^{n+1}}+\frac{\alpha(\xi)(n-1) !}{(\alpha(\xi)-s) t^{n}}\left[a_{1}(\xi)-\frac{s}{2} a_{0}(\xi) n\right]+\ldots
\end{aligned}
$$

Applying Theorem 4, the Donaldson-Futaki invariant is given by

$$
F u t\left(Y_{0}, \xi, \eta\right)=\frac{-1}{n(n+1)}\left[\frac{a_{1}(\xi)}{\alpha(\xi)}-\frac{n(n+1)}{2} a_{0}(\xi)\right] \text {. }
$$

Until now, our developments have been completely general, and equation (14) is the formula for the Donaldson-Futaki invariant of the Rees algebra for a homogeneous principal ideal. We now employ the assumption that the $Y$ is Gorenstein and Calabi-Yau, and $\Theta \in H^{0}\left(X, K_{X}\right)$ is a non-vanishing section satisfying $\mathcal{L}_{\xi} \Theta=i(n+1) \Theta$; equation (12) applies, and so

$$
F u t\left(Y_{0}, \xi, \eta\right)=-\frac{1}{2}\left[\frac{1}{\alpha(\xi)}-1\right] \text {. }
$$


In particular, we have the following theorem, which was proved for rational Reeb vector fields in 32 .

Theorem 7. Let $(Y, \Theta)$ be an isolated Gorenstein singularity with link L, and Reeb vector field $\xi$ satisfying $\mathcal{L}_{\xi} \Theta=i(n+1) \Theta$. If $Y$ admits a holomorphic function $f$ with $\mathcal{L}_{\xi} f=i \lambda f$, and $\lambda<1$, then $(Y, \xi)$ is K-unstable.

This gives the following corollary, which was first observed in [16].

Corollary 4. Let $(Y, \Theta)$ be an isolated Gorenstein singularity with link L, and Reeb vector field $\xi$ satisfying $\mathcal{L}_{\xi} \Theta=i(n+1) \Theta$. If $Y$ admits a holomorphic function $f$ with $\mathcal{L}_{\xi} f=i \lambda f$, and $\lambda<1$, then $(Y, \xi)$ does not admit a compatible constant scalar curvature Kähler metric. In particular, $L$ does not admit a Sasaki-Einstein metric with Reeb field $\xi$.

Note that even if $Y$ is not a Gorenstein singularity, from (14) we obtain a lower bound on $\alpha(\xi)$ in terms of the ratio $a_{1} / a_{0}$ whenever $\xi$ is a K-semistable Reeb field on $Y$.

\section{REFERENCES}

[1] B. S. Acharya, J.M. Figueroa-O'Farrill, C. M. Hull, and B. Spence, Branes at conical singularities and holography, Adv. Theor. Math. Phys. 2(1998) 1249-1286.

[2] V. Apostolov, D. M. J. Calderbank, P. Gauduchon, and C. W. Tønnesen-Friedman, Hamiltonian 2-forms in Kähler geometry III, extremal metrics and satbility, Invent. Math., 173(2008), no. 3, 547-601.

[3] T. Aubin, Équations du type Monge-Ampère sur les variétés kählériennes compactes, Bull. Sci. Math. (2) 102 (1978), no. 1, 63-95.

[4] W. L. Baily, On the imbedding of V-manifolds in projective space, Amer. J. Math. 79(1957), 403-430.

[5] C.P. Boyer, K. Galicki and S. R. Simanca, Canonical Sasakian metrics, Comm. Math. Phys., 279 (2008), no. 3, 705-733.

[6] C.P. Boyer and K. Galicki, On Sasakian-Einstein Geometry, Int. J. Math., 11 (2000), 873-909.

[7] C.P. Boyer and K. Galicki, Sasakian Geometry, Oxford University Press, Oxford, 2008.

[8] T. C. Collins, Stability and convergence of the Sasaki-Ricci flow, preprint, arXiv:1105.3947.

[9] S. Donaldson, Lower bounds on the Calabi functional, J. Diff. Geom., 80(2005), no. 3, 453-472.

[10] S. K. Donaldson, Scalar curvature and stability of toric varieties, J. Differential Geom., 62 (2002), 289-349.

[11] A. El-Kacimi Alaoui, Opérateurs transversalement elliptiques sur un feuilletage Riemannien et applications, Compos. Math. 79 (1990), 57-106.

[12] D. Eisenbud, Commutative algebra with a view toward algebraic geometry, SpringerVerlag, New York, 1995.

[13] A. Futaki and H. Ono, Einstein metrics and GIT stability, Sugaku Expositions 24 (2011), no.1, 93-122.

[14] A. Futaki, H. Ono and Y. Sano, Hilbert series and obstructions to asymptotic semistability, Adv. Math. 226 (2011), no. 1, 254-284.

[15] A. Futaki, H. Ono and G. Wang, Transverse Kähler geometry of Sasaki manifolds and toric Sasaki-Einstein manifolds, J. Differential Geom., 83 (2009), no.3, 585-636. 
[16] J.P. Gauntlett, D. Martelli, J. Sparks, S.-T. Yau, Obstructions to the existence of Sasaki-Einstein metrics, Comm. Math. Phys. 273 (2007), no.3, 803-827.

[17] W. He and S. Sun, Frankel conjecture and Sasaki geometry, arXiv:1202.2589

[18] T. Kawasaki, The Riemann-Roch theorem for complex V-manifolds, Osaka J. Math., 16 (1979), no. 1, 151-159.

[19] A. Kehagias, New type IIB vacua and their F-theory interpretation, Phys. Lett. B 435 (1998) 337-342.

[20] I.R. Klebanov and E. Witten, Superconformal field theory on threebranes at a CalabiYau singularity, Nucl. Phys. B 536(1998) 199-218.

[21] M. Kreuzer and L. Robbiano, Computational Commutative Algebra 2, Spinger-Verlag, Berlin, 2005.

[22] T. Mabuchi, A stronger concept of K-stability, preprint, arXiv: 0910.4617.

[23] T. Mabuchi, K-stability of constant scalar curvature polarization, arXiv:0812.4093.

[24] J. M. Maldacena, The large $N$ limit of superconformal field theories and supergravity Adv. Theor. Math. Phys. 2 (1998), no.2, 231-252.

[25] D. Martelli, J. Sparks and S.-T. Yau, Sasaki-Einstein manifolds and volume minimisation, Comm. Math. Phys. 280 (2008), no.3, 611-673.

[26] D. Martelli, J. Sparks and S.-T. Yau, The geometric dual of a-maximisation for toric Sasaki-Einstein manifolds, Comm. Math. Phys. 268 (2006), no.1, 39-65.

[27] D. R. Morrison, and M. R. Plesser, Non-sphereical horizons. I, Adv. Theor. Math. Phys. 3 (1998) 1-81.

[28] D. Phong, and J. Sturm, Lectures on stability and constant scalar curvature, Current Developments in Mathematics, 2007(2009), 101-176.

[29] D. Phong, and J. Sturm, On stability and the convergence of the Kähler-Ricci flow, J. Differential Geom., 72(2006), no.1, 149-168.

[30] D. Phong, J, Song, J, Sturm, and B. Weinkove, The Kähler-Ricci flow and the $\bar{\partial}$ operator on vector fields, J. Differential Geom., 81 (2009), no. 3, 631-647

[31] J. Ross and R.P. Thomas, A study of the Hilbert-Mumford criterion for the stability of projective varieties, J. Algebraic. Geom. 16(2007), 201-255.

[32] J. Ross and R.P. Thomas, Weighted projective embeddings, stability of orbifolds and constant scalar curvature Kähler metrics, J. Diff. Geom., 88 (2011), no. 1, 109-160.

[33] P. Rukimbira, Chern-Hamilton's conjecture and K-contactness, Houston J. Math., 21 (1995), no.4, 709-718.

[34] K. Smoczyk, G. Wang and Y. Zhang, The Sasaki-Ricci Flow, Inter. J. of Math., 21 (2010), no.7, 951-969.

[35] J. Sparks, Sasaki-Einstein manifolds, Surveys in Differential Geometry 16 (2011), 265-324.

[36] J. Stoppa, K-stability of constant scalar curvature Kähler manifolds, Adv. Math., 221 (2009), no. 4, 1397-1408.

[37] G. Székelyhidi, Extremal metrics and K-stability, Bull. Lond. Math. Soc., 39 (2007), no. $1,76-84$.

[38] G. Tian, Kähler-Einstein metrics with positive scalar curvature, Invent. Math., 137 (1997), no. 1, 1-37.

[39] B. Toën, Théorèms de Riemann-Roch pour les champs de Deligne-Mumford, KTheory, 18 (1999), no. 1, 33-76.

[40] S.-T. Yau, On the Ricci curvature of a compact Kähler manifold and the complex Monge-Ampère equation, I, Comm. Pure. Appl. Math. 31(1978), 339-411.

[41] S.-T. Yau, Open problems in geometry, Proc. Symp. Pure Math., 54 (1993), 1-18. 